University of Nebraska - Lincoln DigitalCommons@University of Nebraska - Lincoln

Xia Hong Publications

Research Papers in Physics and Astronomy

2006

\title{
Planar Hall-effect magnetic random access memory
}

Y.Bason

L. Klein

J.-B. Yau

X. Hong

J. Hoffman

See next page for additional authors

Follow this and additional works at: https://digitalcommons.unl.edu/physicshong

Part of the Atomic, Molecular and Optical Physics Commons, and the Engineering Physics Commons

This Article is brought to you for free and open access by the Research Papers in Physics and Astronomy at DigitalCommons@University of Nebraska Lincoln. It has been accepted for inclusion in Xia Hong Publications by an authorized administrator of DigitalCommons@University of Nebraska Lincoln. 
Authors

Y. Bason, L. Klein, J. -B. Yau, X. Hong, J. Hoffman, and C. H. Ahn 


\title{
Planar Hall-effect magnetic random access memory
}

\author{
Y. Bason ${ }^{a)}$ and L. Klein \\ Department of Physics, Bar Ilan University, Ramat Gan 52900, Israel \\ J.-B. Yau, X. Hong, J. Hoffman, and C. H. Ahn \\ Department of Applied Physics, Yale University, New Haven, Connecticut 06520-8284
}

(Presented on 3 November 2005; published online 18 April 2006)

\begin{abstract}
We suggest a type of magnetic random access memory (MRAM) that is based on the phenomenon of the planar Hall effect (PHE) in magnetic films, and we demonstrate this idea with manganite films. The PHE-MRAM is structurally simpler than the currently developed MRAM that is based on magnetoresistance tunnel junctions, with the tunnel junction structure being replaced by a single-layer film. (C) 2006 American Institute of Physics. [DOI: 10.1063/1.2162824]
\end{abstract}

Among various technologies considered for future memory applications, magnetic random access memory (MRAM) has attractive properties, since in addition to being nonvolatile with high endurance it can also be as fast as static random access memory (SRAM) and as dense as dynamic random access memory (DRAM).

Storing data in a typical MRAM device is accomplished by applying a magnetic field and causing a magnetic layer in the device to be magnetized in one of two possible states. Reading the data stored in a MRAM device requires reading the electrical resistance of the device, which depends on the magnetization orientation. Currently developed MRAM devices are based on magnetoresistance tunnel junctions ${ }^{1,2}$ (MTJ), which are comprised of two ferromagnetic layers separated by a thin, electrically insulating tunnel barrier layer. The operative effect in MTJ structures exploits the asymmetry in the density of states of the majority and minority energy bands in a ferromagnet, with the tunneling resistance depending on the relative orientation of the magnetization vectors in the two magnetic layers. In the parallel configuration, there is a maximal match between the occupied states in one layer and the available states in the other layer, leading to a minimum in the tunneling resistance.

Current MTJ structures can achieve $\sim 200 \%$ (Refs. 3 and 4) resistance differences between the parallel and antiparallel magnetization configurations, but these structures require a layering of numerous films and a relatively precise control of the thickness of the insulating layer. Also, in both states the measured voltage is of the same sign, so if MTJ structures are used in arrays, the variance of the voltages in the array must be much smaller than the difference in the average values of the distribution of the two states in the array.

We suggest here a different approach for storing a magnetic bit, which is based on the planar Hall effect (PHE). ${ }^{5}$ The PHE in magnetic conductors occurs when the resistivity depends on the angle between the current density $\mathbf{J}$ and the magnetization $\mathbf{M}$, an effect known as anisotropic magnetoresistance (AMR). ${ }^{6}$ The AMR yields a transverse electric field when $\mathbf{J}$ is not parallel or perpendicular to $\mathbf{M}$. If we assume $\mathbf{J}$

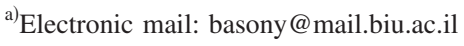

in the $x$ direction and $\mathbf{M}$ in the $x-y$ plane with an angle $\theta$ between them, the generated electric field has both a longitudinal component,

$$
E_{x}=\rho_{\perp} j_{x}+\left(\rho_{\|}-\rho_{\perp}\right) j_{x} \cos ^{2} \theta,
$$

and a transverse component,

$$
E_{y}=\left(\rho_{\|}-\rho_{\perp}\right) j_{x} \sin \theta \cos \theta .
$$

The latter component is denoted the planar Hall effect. Unlike the ordinary and extraordinary Hall effects, the PHE shows an even response upon inversion of $\mathbf{J}$ and $\mathbf{M}$. Therefore, the PHE is most noticeable when $\mathbf{M}$ changes its axis of orientation, in particular between $\theta=45^{\circ}$ and $\theta=135^{\circ}$. The PHE in magnetic materials has been previously investigated in $3 \mathrm{~d}$ ferromagnetic metals, such as Fe, Co, and Ni films, as a tool to study in-plane magnetization. ${ }^{7}$ It has also been studied for low-field magnetic sensor applications. ${ }^{8}$ Recently, large resistance jumps in the PHE have been discovered in the magnetic semiconductor $\mathrm{Ga}(\mathrm{Mn})$ As below its Curie temperature, $\sim 50 \mathrm{~K}$. Four orders of magnitude larger than what has been observed in ferromagnetic metals, it has been termed the giant planar Hall effect (GPHE). ${ }^{9}$ We previously reported that the GPHE can be observed in thin manganite films ${ }^{10}$ at temperatures up to $\sim 140 \mathrm{~K}$. Here we show the PHE in manganite films above room temperature. In addition, we demonstrate the possible use of a thin manganite film as a memory cell operating at room temperature.

The samples we use are epitaxial thin films $(\sim 35 \mathrm{~nm})$ of $\mathrm{La}_{1-x} \mathrm{Sr}_{x} \mathrm{MnO}_{3}$ (LSMO), with $x=0.35$, and a resistivity peak temperature of $\sim 390 \mathrm{~K}$ (see Fig. 1). The films are patterned for longitudinal and transverse resistivity measurements, with the current path along the [100] and [010] directions. Voltage sensing leads are connected perpendicular to the current path in a "Hall-like" configuration (see inset of Fig. 1). The "active area" (between $C$ and $D$ ) on the current path is $2 \times 2 \mu \mathrm{m}^{2}$.

Magnetizing the film along $\theta=45^{\circ}$ and $\theta=135^{\circ}$ yields a PHE with opposite signs, according to Eq. (2), which can serve as the two states of a memory cell. Figure 2 shows the PHE signal, $R_{C D}$, defined as the voltage between $C$ and $D$ divided by the current between $A$ and $B$, while a field of 50 Oe is applied and then removed along $\theta=45^{\circ}$ and $\theta=135^{\circ}$, at 


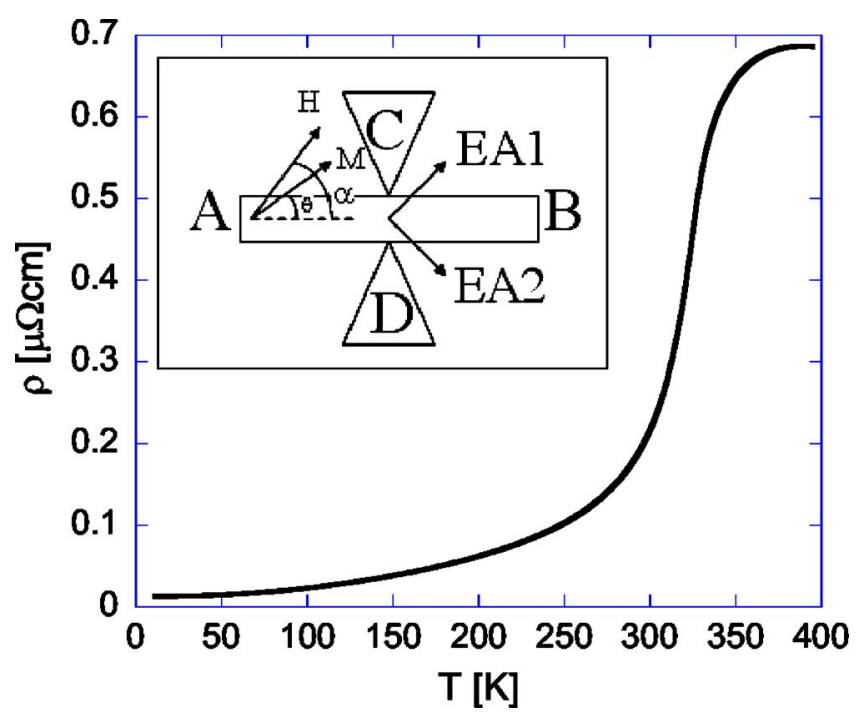

FIG. 1. Resistivity vs temperature of an epitaxial LSMO sample. The peak temperature is at $\sim 390 \mathrm{~K}$. Inset: The pattern used for the experiments. The two easy axes are EA1 and EA2. The angle between the applied magnetic field $\mathbf{H}$ and the current path is denoted $\alpha$, and the angle between the magnetization $\mathbf{M}$ and the current path is denoted $\theta$.

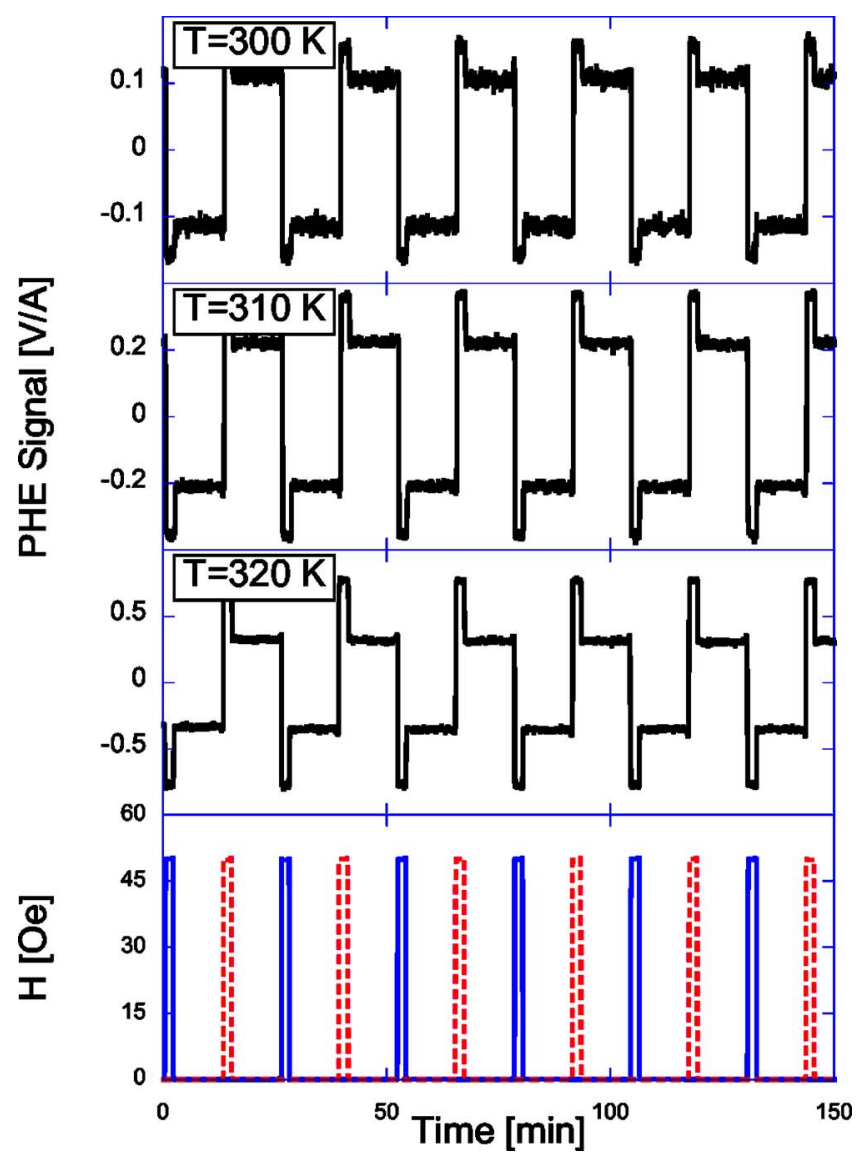

FIG. 2. Planar Hall signal in a LSMO film vs time at different temperatures. A 50 Oe magnetic field is applied parallel to one of the easy axes (EA2). The field aligns the magnetization along this axis, and a negative signal is observed. The magnetic field is then turned off, leaving the magnetization in its remanent state along this axis. After $10 \mathrm{~min}$, the magnetic field is pulsed on and then off, this time parallel to the other easy axis, which leaves the magnetization in remanent state along EA1. This leads to a positive signal reading. This procedure is repeated several times. The bottom graph shows the magnitudes of the magnetic field directed along EA1 (dashed line) and the magnitudes of the magnetic field directed along EA2 (solid line).

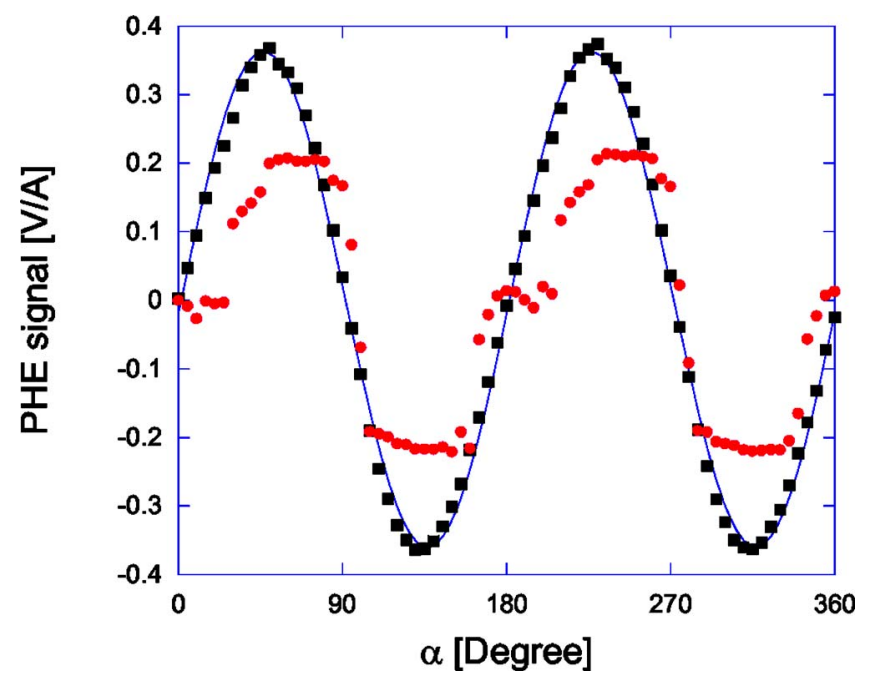

FIG. 3. Planar Hall signal as a function of $\alpha$ with (squares) and without (circles) an applied 50 Oe magnetic field directed at $\alpha$ with respect to the current path. The line connecting the squares is a fit to Eq. (2).

temperatures of 300,310 , and $320 \mathrm{~K}$. The temperature dependence of the transverse voltage shows that the signal increases with temperature up to $T=320 \mathrm{~K}$. In the lowtemperature regime, the effect is measurable down to $T$ $=270 \mathrm{~K}$. We see that the two memory states are clearly separated and stable in time.

We notice that the PHE signal decreases in absolute value when the field is removed. To explore the origin of this behavior we measured $R_{C D}$ with and without magnetic field as a function of $\alpha$, the angle between the magnetic field and the current (Fig. 3). The applied field (50 Oe) is bigger than the coercive field of the sample. Therefore, when the field is on, the magnetization is parallel to the applied field and $R_{C D}$ follows the behavior expected from Eq. (2). In the absence of the magnetic field, we see that $R_{C D}$ has several plateaus, which we attribute to biaxial magnetocrystalline anisotropy with easy axes along $\theta \sim 75^{\circ}$ and $\theta \sim 105^{\circ}$, combined with shape anisotropy along the current path. The role of shape anisotropy was revealed by measuring patterns with current paths in different orientations. While the effect of these anisotropies decreases the observed signal, the results presented in Fig. 2 indicate that the two magnetic states are still well separated.

The results presented here demonstrate the potential of using the PHE as the basis for a new type of MRAM. The device possesses structural simplicity (a single-layer thin film compared to a tunnel junction), and the measured voltages involve a sign reversal between the two states.

One of the authors (L.K.) acknowledges support by Intel-Israel and by the Israel Science Foundation founded by the Israel Academy of Sciences and Humanities. He and another author (C.A.) acknowledge support from the United States - Israel Binational Science Foundation (BSF), Jerusalem, Israel (Grant No. 2002384).

${ }^{1}$ J. S. Moodera, L. R. Kinder, T. M. Wong, and R. Meservey, Phys. Rev. Lett. 74, 3273 (1995).

${ }^{2}$ T. Miyazaki and N. Tezuka, J. Magn. Magn. Mater. 151, 403 (1995).

${ }^{3}$ S. S. P. Parkin, C. Kaiser, A. Panchula, P. M. Rice, B. Hughes, M. Samant, 
and S.-H. Yang, Nat. Mater. 3, 862 (2004).

${ }^{4}$ S. Yuasa, T. Nagahama, A. Fukushima, Y. Suzuki, and K. Ando, Nat. Mater. 3, 868 (2004).

${ }^{5}$ C. Goldberg and R. E. Davis, Phys. Rev. 94, 1121 (1954); F. G. West, J. Appl. Phys. 34, 1171 (1963); W. M. Bullis, Phys. Rev. 109, 292 (1958).

${ }^{6}$ T. R. McGuire and R. I. Potter, IEEE Trans. Magn. 11, 1018 (1975).

${ }^{7}$ B. Zhao, X. Yan, and A. B. Pakhomov, J. Appl. Phys. 81, 5527 (1997); A.

Nemoto, Y. Otani, S. G. Kim, K. Fukamichi, O. Kitakami, and Y. Shimada Appl. Phys. Lett. 74, 4026 (1999); G. Li, T. Yang, Q. Hu, H. Jiang, and W.
Lai, Phys. Rev. B 65, 134421 (2002); Z. Q. Lu and G. Pan, Appl. Phys. Lett. 80, 3156 (2002); S. Das, H. Yoshikawa, and S. Nakagawa, J. Appl. Phys. 93, 8098 (2003).

${ }^{8}$ A. Schuhl, F. Nguyen Van Dau, and J. R. Childress, Appl. Phys. Lett. 66, 2751 (1995).

${ }^{9}$ H. X. Tang, R. K. Kawakami, D. D. Awschalom, and M. L. Roukes, Phys. Rev. Lett. 90, 107201 (2003).

${ }^{10}$ Y. Bason, L. Klein, J.-B. Yau, X. Hong, and C. H. Ahn, Appl. Phys. Lett. 84, 2593 (2004). 